\title{
Atrial septal defect (ASD) device closure - the revolution continues
}

\author{
Galappatthy $\mathbf{G}^{1}$
}

Journal of the Ceylon College of Physicians, 2016, 47, 8-14

President and Council of Ceylon College of Physicians, President and Council of Kandy Society of Medicine, ladies and gentlemen,

I thank the Ceylon College of Physicians for giving me this opportunity to deliver the prestigious Dr. Cyril Fernando memorial oration at the $4^{\text {th }}$ Foundation Sessions of the CCP this evening in Kandy. I am delighted to be back among my dear friends and colleagues of the KSM, to which I belonged from 2004 to 2007, as Resident Cardiologist, Teaching Hospital, Kandy.

Dr. Cyril Francis Fernando, C.M.G., O.B.E., M.D., M.R.C.P. was a Sri Lankan clinician and researcher. Born in 1900 in Colombo, he was educated at St. Benedict's College, Colombo that boasts of alumni such as the revered Anagarika Dharmapala and Narada Thero among others. He attended the University College Hospital, London, qualifying M.B. B.S. in 1925 and obtained MRCP in 1926 and M.D. in 1929, winning the gold medal for the best performance in the MD examination. In 1929, he was appointed physician to the outpatients of Colombo General Hospital and rose steadily in the visiting staff, and serving this institution for the next 25 years. He was an external faculty member and examiner in medicine in the then Ceylon Medical College and later the Faculty of Medicine, University of Ceylon. He was honorary secretary and treasurer 1930-32 of the Ceylon Branch of the British Medical Association (later renamed the Sri Lanka Medical Association), vice-president in 1942, and president in 1948-9. His son Dr. Malik Fernando also served as the President of the Sri Lanka Medical Association in 2012. Dr. Cyril Fernando was awarded the O.B.E. in 1949 and C.M.G. in 1955 the year he passed away.

I feel the following extract from a 1955 BMJ obituary for Dr. Cyril Fernando which quotes an appreciation written by Professor G H Cooray - a contemporary of his, gives us insight into this unique gentleman we are honoring today.

\footnotetext{
${ }^{1}$ Consultant Cardiologist, National Hospital of Sri Lanka.
}

E-mail: gamini_galappatthy@hotmail.com

\begin{abstract}
"As a teacher Cyril Fernando was loved and respected by his students who will miss his pronouncements expressed with such clarity and precision. His sayings were often tinged with wit and became aphorisms among students of the medical faculty. To the bedside he brought the benefit of a wide experience of disease, and understanding of human nature. To his patients who were drawn from the poorest as well as the richest of the land he was equally kind and sympathetic. Cyril, as he was known to his friends and colleagues inspired such confidence that rarely was a diagnostic complexity, either in the hospital wards or in private nursing wards, cleared up without his co-operation. Although he reached the top of the profession he never forgot his modest beginnings, and very recently he donated a science laboratory to the school where he received his elementary education."
\end{abstract}

Closer to my heart I was excited to find that Dr. Fernando has reported the first documented cases of ischaemic heart disease in Sri Lanka in a 1939 publication titled "two cases of coronary disease in early life" in the Journal of the Ceylon branch of the British Medical Association. This is mentioned by Dr. Shanthi Mendis when delivering this same Cyril Fernando memorial oration in 1991.

The Ceylon College of Physicians commemorates the memory of this eminent physician annually with the award of a prestigious Gold Medal Oration, and I am greatly honored to deliver this year's Dr. Cyril Fernando memorial oration on "Atrial septal defect (ASD) device closure - the revolution continues" describing my single operator experience of device closing over 600 ASDs successfully in Sri Lankan patients and seven associated papers, six of which were presented at international fora.

\section{Introduction}

The estimated prevalence of ASD worldwide is about 1 per 1000 live births. ${ }^{1}$ In Sri Lanka with a little over 100,000 live births per year, we can therefore expect about 100 new ASDs to be added annually. Given a life expectancy of an average of 70 years, a total of over 6000-7000 ASDs are likely to be present in the entire country; with a large number of these yet to be diagnosed. Every year about 400 of these would undergo ASD closure. Device closure will thus continue 
to play an important role for many years to come in the management of secundum type ASDs in children and adults.

\section{Aim}

To describe the outcome of 706 consecutive ASD device closures done by a single operator during 89 months from March 2007 to July 2014.

\section{Evolution of a modification of a standard method}

Trans-catheter device closure of ASD was first attempted by King and Mills in 1976 and soon became an effective and safe alternative to surgery. It was accepted as the gold standard of therapy for closure of all suitable secundum ASD at the beginning of the millennium. ${ }^{2}$ ASD closure hitherto involved open-heart surgery lasting several hours with cardio pulmonary bypass. The advent of this technique has offered a great advantage in reduced cost, hospital stay and surgical morbidity to patients. Moreover it saves valuable operating time of cardio thoracic surgeons burdened with long operation lists. Percutaneous device closure of ASD thus revolutionized the practice of ASD closure.

Several types of ASD devices are in use today. Those which stent the ASD with the waist of the device are the nitinol double disc devices such as the Amplatzer septal occluder. Those which patch-close the ASD are the Cardioseal and Gore Helix which cannot be used to close the larger ASDs. The device gets covered by endothelium usually by six months as shown in a study of a series of explanted devices from animal studies. The Amplatzer type has shown the least risk of thrombus formation on device in trials. In Sri Lanka since the first device closure done around 2003 , we use this type of device which costs approximately US\$800 each.

The Amplatzer type double disc septal occluder consists of two discs connected by a waist and is woven with nitinol a nickel - titanium alloy wire which gives shape memory to the device to self-expand. ${ }^{3}$ The device size is determined by the diameter of the waist and 4-42 $\mathrm{mm}$ devices are available for closing ASDs with a diameter of up to about $36 \mathrm{~mm}){ }^{3}$ The waist stents and holds to the ASD, while the discs seal the rim supporting the device. A layer of nylon sewn inside the device occludes the left to right shunt blood flow across the ASD. Delivered inside a 9-12 French size catheter placed across the ASD the device is deployed on either side of the ASD. This core technique is as follows. ${ }^{3}$ The device is screwed on to a delivery cable and loaded into a delivery sheath. The sheath is advanced through the femoral vein up the inferior vena cava and positioned across the ASD under fluoroscopy guidance. When the device is pushed out of the delivery sheath, first the left atrial disc expands, followed by the waist stenting the ASD and next the right atrial disc expands. After confirming the correct positioning and stability, the cable is unscrewed and removed, thereby releasing the device. If the device position is unsatisfactory before release the device can be repositioned or exchanged. Retrieval of a released device using a snare is also possible percutaneously through the delivery sheath. The challenge for the operator is to select the appropriate device size and to skillfully place it across the ASD. Correct sizing of the device to match the ASD is crucial for procedure success as too small a device tends to embolize and too big a device tends to be bulky after implantation. Varying techniques are used by operators worldwide to achieve this.

Device closure is done as a sterile procedure in a cardiac catheterization laboratory using fluoroscopy. The standard method as done in the USA and most countries, is usually performed under local anesthesia, but under general anesthesia by some, involves inflating a sizing balloon inside the ASD to measure the 'stop flow' or stretched ASD diameter and selecting a device with a waist of similar diameter. ${ }^{2,4}$ Assessment of device positioning and profile is done relying on invasive transoesophageal (TOE) or by a more costly intra cardiac echocardiography (ICE). All these steps add their own risks, costs and time to the overall procedure. The sizing balloon costs approximately US $\$ 600$ and the procedure costs about US\$7000 in USA without the device cost. Device closure done by this standard method took up to 90 minutes as reported in a Sri Lankan paper by Rajakanthan $\mathrm{K}$ and co workers titled "Device closure of atrial septal defects - initial experience in over 200 patients" presented at the 2007 SLMA annual sessions. ${ }^{5}$.

In 2008, we introduced a novel safe and simplified modified technique with less procedural steps. From an audit of the initial 70 ASD device closures done for balloon sizing by the above mentioned standard technique, we derived a device sizing formula by adding 4-6 $\mathrm{mm}$ to the maximum ASD diameter measured by transthoracic echocardiography (TTE), to select the appropriate device waist size. This roughly corresponds to the likely stretching of the ASD by the sizing balloon inflation. Next, we determined three transthoracic echo views to assess the ASD rim nearly in its entirety, namely the modified 4 chamber view to assess the mitral margin, parasternal short axis view to assess the antero-posterior margin and the epigastric view to assess the superior inferior margin as shown in Figure 1. Transcaval margins crucial to device stability is usually assessed by TOE; but was assessed by us in the transthoracic epigastric view in the superior inferior plane by axially rotating the probe clockwise for the IVC margin and anticlockwise for the SVC margin. 
These three views were used during the procedure to assess optimum device positioning. When the device is correctly positioned as shown in Figure 2, these views clearly show the double disk device held across the ASD by the waist with the two discs on either side of the septum had no colour Doppler flow across. We did not encounter difficulty in using the epigastric view in adult patients. With these three reliable TTE images matching the defect- device size estimated by our own sizing formula, invasive TOE and balloon sizing became obsolete and were thus excluded from our protocol. Therefore the modified technique introduced by us, simply consists of positioning the delivery sheath across the ASD, and deploying the pre-selected device under fluoroscopy, relying on fluoroscopy appearance of the device turning anteriorly to indicate correct alignment of the device parallel to the septum, and confirming optimum device positioning by TTE using the above mentioned three views.

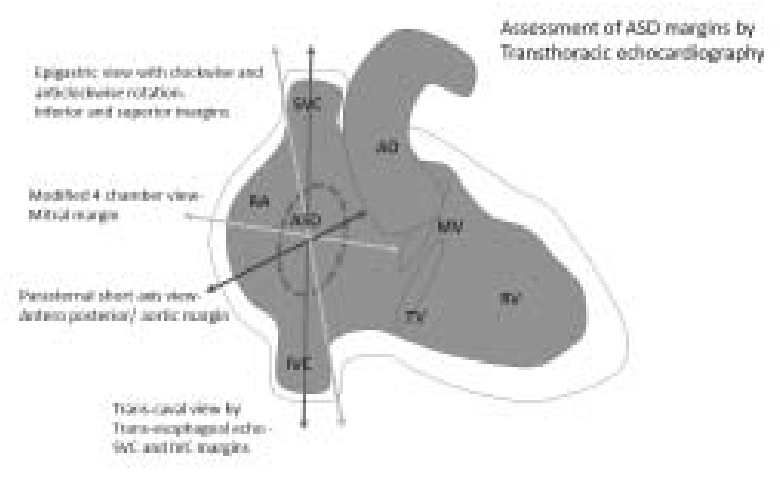

Figure 1. Schematic diagram of ASD margins seen in echocardiography views.

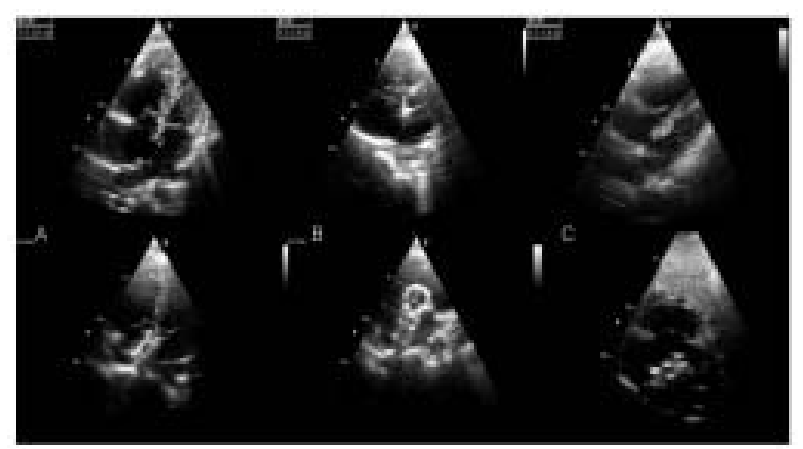

Figure 2. ASD assessment using transthoracic echocardiography: A. Modified 4 chamber. B. parasternal short axis and C. epigastric views. Top row assessing ASD size and margins in A- mitral margin, in B- antero posterior margin and in $\mathrm{C}$ - superior inferior margin. Bottom row assessing ASD device position in same views.
In 2009, we presented for the first time this modified method in a paper titled "Outcome of device closure of atrial septal defects (asds) using a modified technique" at the SLMA $141^{\text {st }}$ Annual Academic Sessions, ${ }^{6}$, which was awarded the SLMA special prize for cardiology that year. This study analyzed 251 ASD patients done by both techniques and showed the modified method was safe, cost effective, with low radiation exposure for the patient and physician, and enabled fast procedural times of on average 15 minutes. Using this innovative, user friendly and efficient technique which became widely accepted and adopted by other cardiologists, the number of procedures done at the NHSL jumped many fold to the present number of approximately 300 cases annually, and a total in excess of 2000 cases done to date at the NHSL; thus further revolutionizing the practice of ASD closure in Sri Lanka.

In 2013 a paper titled "Outcome of 511 atrial septal defect device closures without balloon sizing" analyzing 511 ASD device closures done solely by the modified technique with no balloon sizing was presented to the Society for Cardiac Angiography and Intervention (SCAI) conference in Orlando, USA in March 2013, concluding the feasibility, safety and cost effectiveness of this method. ${ }^{7}$ A similar paper titled "Outcome of 511 atrial septal defect device closures without balloon sizing or routine invasive echocardiography" that additionally highlights the trans thoracic echo assessments was presented to the Congenital Structural Intervention (CSi) conference in Frankfurt, Germany in May 2013 to share this innovative technique with our international peers. ${ }^{8}$

A cost analysis of the same data was presented to the WHO 2nd Global Devices Forum 2013 in Geneva titled "Atrial septal defect (ASD) device closure without invasive echocardiography and using a sizing formula instead of balloon sizing is safe cost effective and increases access to treatment."9 Approximate cost of the procedure excluding device cost was estimated to be US $\$ 300$ by the modified method versus. US $\$ 7000$ by the standard technique. This study highlighted the safety and cost saving of the modified technique and concluded that the modified technique increases access to treatment for patients in low socio economic settings, by virtue of the significant cost savings and reduced procedure times.

\section{Methodology}

Inclusion and exclusion criteria - As per standard guidelines $(1,10)$, TTE or TOE confirmed secundum ASDs $>6 \mathrm{~mm}$ with a left to right shunt with an adequate rim $>5 \mathrm{~mm}$, and fenestrated ASD (FASD) or persistent foramen ovale (PFO) with likely paradoxical embolism through the defect were included for device closure. 
Exclusion criteria were ASD $>38 \mathrm{~mm}$, deficient inferior margin, severe pulmonary hypertension with absent left to right shunt, more than one anomalous pulmonary vein draining into the right atrium, primum ASD, sinus venosus ASD and declining the modified method by the patient.

Consent - Informed written consent was obtained prior to all invasive procedures.

Statistical analysis - Data from a single operator who performed ASD device closure registry on a Microsoft Office 2007-Excel and Apple Numbers program were analyzed. 'GraphPad InStat version 3.06' was used for statistical calculations.

Funding - The cost of the ASD devices were funded by the Ministry of Health [in hospital stock] or the President's fund in most cases or by the patient. Six brands of Amplatzer type devices registered in Sri Lanka were used each priced at Sri Lankan Rupees 150,000 .

Medications - Aspirin $300 \mathrm{mg}$ orally was given the night before and heparin intravenous bolus of $50-75$ units $/ \mathrm{kg}$ was given at the start of the procedure. Intravenous cefuroxime $750 \mathrm{mg}$ was given at the commencement and $500 \mathrm{mg}$ orally twice daily for three doses thereafter. Aspirin low dose was continued for 6-12 months after successful device closure. Clopidogrel $75 \mathrm{mg}$ was added for those with symptoms suggestive of migraine and pantoprazole orally for symptoms of gastritis.

Technique - ASD device closure was done as per local protocols in the cardiac catheterization laboratory with fluoroscopy, under local anesthesia, percutaneously through right femoral vein approach. Meticulous attention to prevent air embolism such as flushing all catheters and underwater device loading was ensured.

The device closure was performed according to the standard technique with balloon sizing carried out in the initial 70 patients. The modified technique as described above using a device sizing formula and no invasive echocardiogram was done in the subsequent ASD patients. FASD and PFO closure techniques were largely similar to the modified technique with custom devices that were sized by different sizing formulae and were assessed by real time 3-dimensional (RT3D) trans oesophageal echocardiogram.

Follow up - Outpatient follow up was at 2 weeks, 6 months and annually thereafter with transthoracic echocardiography assessment at each visit.

\section{Results and discussion}

A total of 706 consecutive ASD device closure procedures was analyzed - 485 were females (69\%). Mean age 33 years ( $S D=16$, range $7-76$ years). Thirty two were below 12 years.

Of these, 649 patients were successfully device closed (initial 70 by the standard technique with balloon sizing, 556 by the modified technique and 22 fenestrated ASD closures). Acute procedure success defined as good device position and profile without residual shunts up to time of discharge (usually 2nd day) was $92 \%$. Majority of the failures were aborted procedures due to unfavorable ASD anatomy. The procedural success rate of $92 \%$ is similar to other studies with balloon sizing (85\%-97\%) and could be improved by stricter patient selection.

Mean ASD diameter was $20 \mathrm{~mm}(\mathrm{SD}=6$, range 6$36 \mathrm{~mm})$. Mean device size was $24 \mathrm{~mm}(\mathrm{SD}=6$, range 8-36 mm). Mean over sizing to maximum transthoracic echo ASD diameter was $4 \mathrm{~mm}$ (range 2-10 mm). Up or down-sizing of device was done in 32 patients with large ASD (5\%).

Average procedure time was 15 minutes by the modified method. Mean number of device closures done in a routine mixed catheter list was 3 (range 1-12) per session. The estimated procedure cost by this method excluding device cost is about US\$300.

Echocardiography pictures of the ASD before and after device closure and during follow up show changes in echo appearance of the device due to ongoing covering of the device by endocardium with fibrosis and cardiac remodeling.

Minor complications occurred in 34(5\%), which includes air embolism to the right coronary artery, ${ }^{4}$ thromboembolism to left coronary artery, ${ }^{1}$ device embolism $^{8}$ retrieved percutaneously in 5 and surgically in 3, Dressler's syndrome, ${ }^{3}$ pulmonary edema ${ }^{1}$ in a patient with moderate mitral regurgitation, bleeding from puncture site ${ }^{2}$ and transient arrhythmias. ${ }^{15}$ most commonly $\mathrm{AF}^{10}{ }^{10} \mathrm{SVT},{ }^{4}$ and sinus arrest during device positioning $^{1}$ in a patient with a high secundum ASD close to the SVC and SA node.

There were no major complications of deaths, CVA/ TIA, perforations, tamponade, endocarditis or thrombus on device. There were no device erosions (estimated incidence 1 per 1000) in follow up at 1 year (68\% followup) and at 4 years (32\% follow-up). Percutaneous device retrieval during ASD closure was done in 5 patients successfully all from the main pulmonary artery (MPA), and closed with a bigger device. 
These complication rates are comparable to other studies ${ }^{3,4,11}$ with balloon sizing (minor $5 \%$, other studies < $9 \%$; major $0 \%$, other studies $0.3 \%$; erosions $0 \%$, other studies $0 \%-0.1 \%$ ).

The complication of a successful thrombus aspiration from the LAD during ASD device closure was presented as a case report at the American College of Cardiology (ACC) Academic sessions 2012 in Chicago titled "Acute chest pain during percutaneous device closure of an atrial septal defect". ${ }^{12}$ A 42 year old female developed sudden severe chest pain during ASD device positioning with monitor ECG showing ST segment elevation. Coronary artery air or thromboembolism was suspected and a coronary angiogram was promptly performed. Thromboembolism to the left anterior descending coronary artery was diagnosed and primary $\mathrm{PCl}$ with thrombus aspiration was carried out within and establishing complete reperfusion in the left coronary artery thereby preventing a large anterior STEMI. This case report was also presented at the Society for Cardiac Angiography and Intervention (SCAI) sessions titled "Acute chest pain during device closure of an ASD" in Orlando September 2013 and was awarded the 'best save' prize. ${ }^{13}$

There were several interesting patients with coexisting abnormalities who underwent ASD device closure. Three had anomalous pulmonary venous drainage, 1 had bilateral SVC with L- SVC draining to coronary sinus, and 2 had Ebstein's anomaly. ASD device closure was done at the same sitting combined with balloon pulmonary valvuloplasty in 2 patients, with percutaneous trans mitral commisurectomy (PTMC) in 1 , and with stenting to the LAD in 2 patients.

In an illustrative case a 53 y old patient with tight proximal LAD stenosis and a $25 \mathrm{~mm}$ sec ASD with moderate pulmonary hypertension, underwent $\mathrm{PCl}$ to LAD with a drug eluting stent and ASD device closure with a $28 \mathrm{~mm}$ device percutaneously thereafter at the same sitting. Closing the ASD left to right shunt will increase the LV preload on the ischaemic LV with an increased risk of LV failure, and it is therefore important to relieve the ischemia by stenting before closing the ASD.

Interestingly 6 patients with late pericardial patch failure after surgical ASD closure were successfully device closed. The average time after surgery was 14 months. The reason for such patch failure is not well documented in literature. This group posed several unique queries due to the device resting partly on the remaining pericardial patch; such as device instability and risk of device embolism, time taken for tissue growth to cover the device and in the event of an embolized device that is not retrievable percutaneously, the high risk of a re-do open heart surgery. This is an important area that needs further study.
Interestingly there were 22 confirmed FASD patients in this cohort who underwent FASD device closure between April to November 2011. Fenestrated ASD comprise a spectrum of multiple small fenestrations in the fossa ovalis region to larger secundum type ASDs divided by multiple strands or surrounding fenestrations. The surgeons describe these as a 'cobweb' appearance and excise the floppy fenestrated area and close the defect with a pericardial patch. Fenestrated ASD are widely believed to facilitate paradoxical embolism resulting in systemic infarction and ischemia which is considered a reason for their closure, although FASD device closure for the prevention of these complications is not fully determined. FASD with larger defects causing left to right shunting need closing on the merits of the shunt size causing a risk of developing pulmonary hypertension. This is an exciting new vista that needs further exploration and research as the 2010 AHA Stroke guidelines ${ }^{10}$ and ESC Adult Congenital Heart Disease Guidelines Update $2010^{1}$ recommend ASD closure for secondary prevention of stroke but lack specific guidance on fenestrated ASD. Adding to the knowledge base on this interesting association, an analysis of the diagnosis and outcome of these FASD device closure patients was presented to the Congenital Structural Intervention (CSi) con-ference in Frankfurt in June 2014 in a paper titled "Detection and device closure of fenestrated atrial septal defects in young adults with suspected paradoxical embolism."14

\section{Fenestrated ASD}

In patients referred for routine echocardiography, FASD was suspected by trans-thoracic echocardiogram and diagnosed by trans-esophageal approach in the presence of multiple low velocity color Doppler shunts resembling a 'shower head' and confirmed by visualizing the FASD with real time 3-dimensional (RT3D) TOE [Philips iE 33, Netherlands] as shown in Figure 3. Maximum diameter of the FASD was measured and the device disc was oversized by 5-10 mm. The recently introduced Amplatzer Cribriform ASD device has specially designed discs to occlude shunts in the peripheral fenestrations in disc sizes 18 , $25,30,35$ and $40 \mathrm{~mm}$, and a $3 \mathrm{~mm}$ waist across all sizes to place inside small fenestrations. FASD device closure is largely similar to secundum ASD device closure but crossing the fenestrations can be challenging, as well as crossing through a defect in the middle of a cluster of fenestrations to center the device. We used a Judkin right coronary catheter with a Terumo straight tip guide wire to cross the FASD. After positioning the device, size match, centering and absence of shunts was confirmed by TTE/TOE/RT3D TOE. Anti-platelet therapy was given according to guidelines and aspirin continued indefinitely for those with a prior infarct. 
Notably in this cohort of 22 FASD patients, 16 $(72 \%)$ had prior systemic infarcts due to suspected paradoxical embolism (14 cerebral and 2 systemic infarcts). Mean age was 32 years (range 13-64 years), females 13 (60\%). Procedural success was 21 (96\%). Mean FASD diameter was $20 \mathrm{~mm}$ (range 11-30mm). Mean device size was $30 \mathrm{~mm}$ (range $15-35 \mathrm{~mm}$ ). One patient with a large central defect was closed with an ASD occluder. RT3D TOE was useful to visualize defect device size match and closure. There were no major complications. Interestingly no systemic infarctions occurred during follow up of a mean 25 months (range 22-29 months).

During this total experience of over 700 ASD device closure procedures, some useful techniques, tips and tricks were mastered to overcome common problems encountered during device closure such as device slipping, bulky device, partly prolapsed device, deformed device, non-retrievable device and complications. The illustrations in Figure 4 describe some of these tips and tricks useful for device positioning and managing complications. ${ }^{15}$

\section{Conclusions}

Device closure using a sizing formula instead of balloon sizing and TTE instead of invasive imaging is feasible, safe and reduces cost, by using fluoroscopy, with the reduced procedure time thereby increasing access to treatment. The modified technique has a steep learning curve but is simple, practical, efficient and easy to master.

Incidence of young stroke in the cohort of FASD was high. 3D visualization of the FASD helped in diagnosis and device closure. FASD device closure is feasible, safe and appeared effective in preventing systemic infarction during available follow up in young adults with suspected paradoxical embolism.

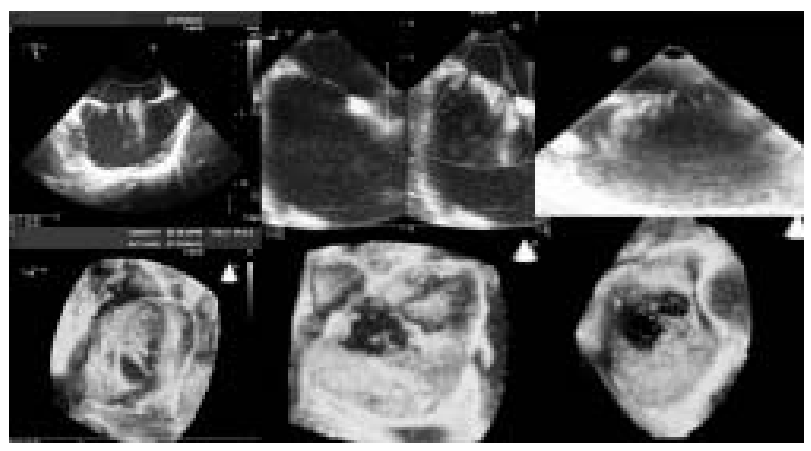

Figure 3. Diagnosing fenestrated atrial septal defects (FASD): Top row shows trans-oesophageal echo images of three patients with FASD showing multiple low velocity colour Doppler shunts resembling a 'shower head'. Bottom row shows real time 3 dimensional transoesophageal (RT3D TOE) images better visualizing the fenestrated ASD.

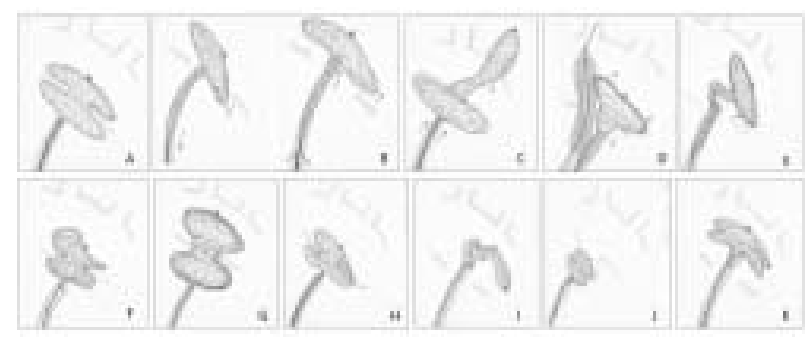

Figure 4. Tips and tricks for device positioning: A. Normal device position with the device waist stenting the ASD and the two discs straddling the atrial septum. B. LA disc slipping inferiorly through the defect corrected by clock wise rotation of device to align the LA disc. C. Device slipping through the defect corrected by deploying the LA disc inside a pulmonary vein, aligning the RA disc parallel to atrial septum and then positioning the LA disc by wiggling the device. D. Aligning a slipping LA disc by inflating a sizing balloon through the ASD. E. Swan neck deformity of the waist is sometimes useful to align the LA disc. F. Bulging LA disk due to device defect need repositioning. G. Both discs are bulging and mushroom shaped due to an oversized device that needs replacement. H. LA disc slipping through the septum needs bigger device or repositioning. I. Damaged device with no shape memory needs replacement. $\mathbf{J}$. When septum is aneurysmal a normally deployed device may mimic a mal-deployed device. K. Both discs in LA and catheter tip invagination prevents device pull back in to the sheath necessitating pull back of the system as a whole.

In summary, ASD device closure was revolutionized in Sri Lanka with the introduction of this modified technique has enabled safe, user friendly and cost effective procedures thereby increasing access to treatment. There is a need to further study the role of FASD in young stroke patients and FASD device closure in the prevention of infarcts. It is now possible to device close ASDs and treat percutaneously some associated abnormalities including coronary artery stenting thus further alleviating the need for open-heart surgery.

And thus the exciting revolution continues...

\section{Acknowledgements}

Dr. Ruvan Ekanayaka - my mentor, and my loving family for their constant encouragement and support, all the staff of the CCP and the Joint Secretaries who I know worked tirelessly to ensure the success of today's event, and a special word of gratitude to my dear friend Dr. Upul Dissanayake who inspired me all along to do this oration. 
All cardiologists and cardiology trainees who facilitated and assisted in numerous ways in these procedures, all physician and neurologist colleagues who referred these patients, and cath lab and ward staff too numerous to mention at NHSL and at Lanka Hospital and Nawaloka Hospital who assisted and continue to do so to make these procedures a success.

Cardio-thoracic surgical colleagues and vascular surgical colleagues who respond promptly to our (rare) complications and without whose safety net none of these procedures would have seen the light of the day.

Last but not the least the innovative industry who are very much a part of this exciting revolution that continues.

\section{References}

1. The Task Force on the Management of Grown-up Congenital Heart Disease of the European Society of Cardiology (ESC). ESC Guidelines for the management of grown-up congenital heart disease (new version 2010). www.escardio.org/ guidelines.

2. Francisco Garay, Qui Ling Cao, Ziyad Hijazi, Device closure of secundum atrial septal defects: in Antonio Colombo, Goran Stankovic Eds, Problem Oriented Approaches in Interventional Cardiology, 2007 Informa UK Ltd.

3. Amplatzer Septal Occluder Product Monograph. AGAMedical Corporation USA, 2004.

4. Fischer G, Steish J, Uebing A, et al. Experience with transcatheter closure of secundum atrial septal defects using the Amplatzer septal occluder: a single centre study in 236 consecutive patients. Heart 2003; 89: 199-204.

5. Rajakanthan K, Arulnithy K, Kamaladasa K, et al. Device closure of atrial septal defects - initial experience in over 200 patients. The Ceylon Medical Journal 2007; 52(S):1-51.

6. Galappatthy GKS, Ponnamperuma CJ, Ekanayake RAI. Outcome of device closure of atrial septal defects [ASDs] using a modified technique. The Ceylon Medical Journal 2009; 54(S):1-22.
7. Galappatthy GKS, Ekanayaka RAI. Outcome of 511 Atrial Septal Defect Device Closures Without Balloon Sizing. Proceedings of the Society for Cardiac Angiography and Intervention (SCAI) conference Orlando March 2013.

8. Galappatthy GKS, Ekanayaka RA I. Outcome of 511 Atrial Septal Defect Device Closures without Balloon Sizing or Routine Invasive Echocardiography. Proceedings of the Congenital Structural Intervention (CSi) conference in Frankfurt May 2013.

9. Galappatthy GK, Ekanayaka RAl. Atrial septal defect (ASD) device closure without invasive echocardiography and using a sizing formula instead of balloon sizing is safe cost effective and increases access to treatment. Abstract No. 168170. Proceedings of the WHO 2nd Global Devices Forum Geneva 2013.

10. Karen L, Furie, Scott E, Kasner, Robert J. Adams, Gregory W, Albers, Ruth L, Bush A, et. al. Guidelines for the Prevention of Stroke in Patients with Stroke or Transient Heart Ischemic Attack: A Guideline for Healthcare Professionals from the American Heart Association/American Stroke Association. Stroke 2011; 42: 227-76.

11. John M, Hegde S, El-Said H, et al. Transcatheter Device Closure of Atrial Septal Defects- A safety review. JACC Cardiovascular Interventions 2013; 6: 433-42.

12. Galappatthy GKS, Ekanayaka RAI. Acute Chest Pain During Percutaneous Device Closure of an Atrial Septal Defect.Proceedings of the American College of Cardiology (ACC) Academic sessions Chicago 2012.

13. Galappatthy GKS, Ekanayaka RAI. Acute Chest Pain During Device Closure of an ASD. Proceedings of the Society for Cardiac Angiography and Intervention (SCAI) annual academic sessions Orlando September 2013.

14. Galappatthy GKS, Ekanayaka RAI. Detection and Device Closure of Fenestrated Atrial Septal Defects in Young Adults with Suspected Paradoxical Embolism. Proceedings of the Congenital Structural Intervention (CSi) conference Frankfurt June 2014.

15. Galappatthy GK . ASD device closure-technique tips and tricks. Proceedings of the 9th Asian Interventional Cardiovascular Therapeutics, Bangkok June 2013. 